\title{
MRI-Based Attenuation Correction for PET/MRI: A Novel Approach Combining Pattern Recognition and Atlas Registration
}

\author{
Matthias Hofmann ${ }^{1-3}$, Florian Steinke ${ }^{2}$, Verena Scheel ${ }^{1}$, Guillaume Charpiat ${ }^{2}$, Jason Farquhar ${ }^{2}$, Philip Aschoff ${ }^{4}$, \\ Michael Brady ${ }^{3}$, Bernhard Schölkopf ${ }^{2}$, and Bernd J. Pichler ${ }^{1}$ \\ ${ }^{I}$ Laboratory for Preclinical Imaging and Imaging Technology of the Werner Siemens-Foundation, Department of Radiology, \\ Eberhard-Karls-University, Tuebingen, Germany; ${ }^{2}$ Max Planck Institute for Biological Cybernetics, Tuebingen, Germany; ${ }^{3}$ Wolfson \\ Medical Vision Laboratory, University of Oxford, Oxford, United Kingdom; and ${ }^{4}$ Department of Radiology, Eberhard-Karls-University, \\ Tuebingen, Germany
}

For quantitative PET information, correction of tissue photon attenuation is mandatory. Generally in conventional PET, the attenuation map is obtained from a transmission scan, which uses a rotating radionuclide source, or from the CT scan in a combined PET/CT scanner. In the case of PET/MRI scanners currently under development, insufficient space for the rotating source exists; the attenuation map can be calculated from the MR image instead. This task is challenging because MR intensities correlate with proton densities and tissue-relaxation properties, rather than with attenuation-related mass density. Methods: We used a combination of local pattern recognition and atlas registration, which captures global variation of anatomy, to predict pseudoCT images from a given MR image. These pseudo-CT images were then used for attenuation correction, as the process would be performed in a PET/CT scanner. Results: For human brain scans, we show on a database of $17 \mathrm{MR} / \mathrm{CT}$ image pairs that our method reliably enables estimation of a pseudo-CT image from the MR image alone. On additional datasets of MRI/PET/ CT triplets of human brain scans, we compare MRI-based attenuation correction with CT-based correction. Our approach enables PET quantification with a mean error of $3.2 \%$ for predefined regions of interest, which we found to be clinically not significant. However, our method is not specific to brain imaging, and we show promising initial results on 1 whole-body animal dataset. Conclusion: This method allows reliable MRI-based attenuation correction for human brain scans. Further work is necessary to validate the method for whole-body imaging.

Key Words: PET/MR; attenuation correction; atlas; machine learning

J Nucl Med 2008; 49:1875-1883

DOI: 10.2967/jnumed.107.049353

\footnotetext{
Received Nov. 26, 2007; revision accepted Jul. 22, 2008.

For correspondence or reprints contact: Bernd J. Pichler, Laboratory for Preclinical Imaging and Imaging Technology of the Werner SiemensFoundation, Röntgenweg 13 72076, Tuebingen, Germany.

E-mail: Bernd.Pichler@med.uni-tuebingen.de

COPYRIGHT @ 2008 by the Society of Nuclear Medicine, Inc.
}

$\mathbf{M}$ ultimodality imaging such as PET/CT, SPECT/CT, and, recently, PET/MRI is an emerging research field. In recent years, the combinations of PET/CT and SPECT/CT have successfully made the transition from basic research into clinical practice. Unfortunately, CT does not provide the excellent soft-tissue contrast that MRI does, adds a significant amount of radiation dose, and does not allow true simultaneous imaging. Therefore, much research in the past few years has concentrated on combining PET and MRI. Although the realization of this combination still poses many technologic challenges, recent progress (1-3) indicates that it is now only a matter of time before PET/MRI scanners will be available for clinical use.

On the software side, attenuation correction (AC), which accounts for radiation-attenuation properties of the tissue, is mandatory to obtain PET images that are sufficiently accurate for quantification. It is also important for visual interpretation and improved lesion detection. Usually in stand-alone PET scanners, the AC is obtained from a transmission scan both with and without the patient, by using 1 or several moving sources (either ${ }^{68} \mathrm{Ge}$ or ${ }^{137} \mathrm{Cs}$ ). In combined PET/CT, AC is derived from the CT information (4). In the case of a PET/ MRI scanner, insufficient space for a rotating source exists, and the attenuation map needs to be determined in another way. Ideally, one would like, as in PET/CT, to obtain the attenuation map directly from the anatomic information, in this case from the MR image.

Predicting attenuation values from MR images is intrinsically difficult because the MRI signal (magnitude and phase) of an individual voxel is related to the proton density, but the signal is not one-to-one related to the electron density information that is needed for $\mathrm{AC}$ of $511 \mathrm{keV}$ of radiation. For example, in most standard MRI sequences, air, compact bone, bed, and coils do not produce any signal, whereas their attenuation coefficients are different. The impossibility of any direct mapping from MRI to CT intensities can also be seen in the joint histogram of these intensities in Figure 1, 
obtained from a human head scan. In particular, for low MRI intensities the same MRI intensity corresponds to a set of different CT values. Similar observations can be made for joint histograms from all standard MRI sequences. The pitfalls of mapping MRI to CT values are also documented in recent work (5). Therefore, standard intensity-based segmentation is bound to fail, and to determine the attenuation value for a given voxel in an MR image we have to include additional knowledge.

There is abundant literature on MR image segmentation. However, the majority of this literature is concerned with the segmentation of the MR image into different types of soft tissue, in particular in the brain. With its high soft-tissue contrast and numerous specialized sequences, MRI is ideal for this purpose. Satisfying results can indeed be achieved with standard segmentation techniques from digital image processing $(6,7)$. Several authors have demonstrated that using neighborhood constraints can improve the results of MRI-based segmentation (8). These methods were developed to distinguish different types of soft tissue. It is unclear whether the methods are helpful for our problem, which includes the need to predict bone structures. Martinez-Möller et al. have argued that bone can be neglected for whole-body imaging (9), but Kops and Herzog show that bone affects the PET reconstruction of brain images, especially in gray matter structures, because of their proximity to the skull (10).

Some authors have presented techniques for segmentation of MR images into air, bone, and soft-tissue classes, which is what is needed for AC purposes (e.g., the review by Zaidi (11)). One of the earliest is from Le Goff-Rougetet et al. (12). El Fakhri et al. $(13,14)$ mention using MRIbased AC but without providing details about implementation or performance evaluation. Zaidi et al. have recently

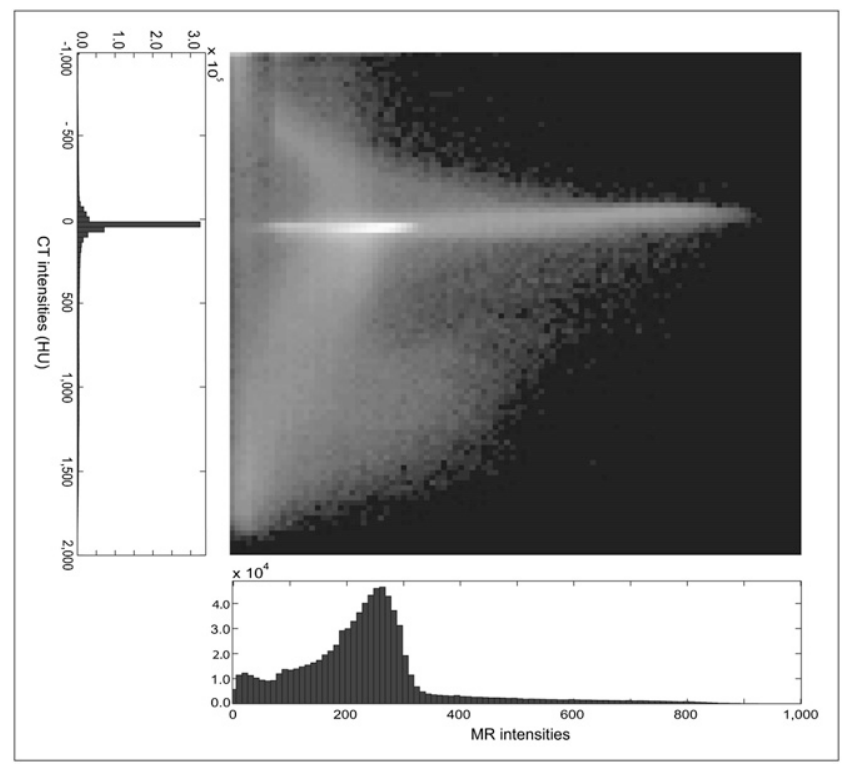

FIGURE 1. 2-dimensional histogram of MRI and CT intensities in T1-weighted head scan. claimed (15) that the method they previously introduced (16) is more robust than the work of El Fakhri et al. and admit that difficulties in the automatic segmentation led to some manual intervention of the operator. Dogdas et al. (17) use a series of morphologic operations to determine soft tissue and bone inside the head and validate their method on a dataset of 8 coregistered MRI/CT pairs. Kops and Herzog (10) evaluate the PET quantification errors of this method. They find that erroneous skull-thickness estimations in Dogdas et al. (17) can lead to errors of up to $10 \%$. Accurate estimation of skull thickness can, therefore, be seen as a necessary requirement for single-digit percentage accuracy. Kops et al. $(10,18)$ have also presented a different MRI template-based AC method, in which they also obtained errors of up to $10 \%$. Robson and Bydder (19) describe an ultrashort echo time MRI sequence, which yields signal from bone. To our knowledge, this has not yet been evaluated with AC.

The approaches mentioned above were developed for application to brain imaging, and the authors do not elaborate on whether the methods would generalize to whole-body application. Template-deformation methods such as those reported by Kops and Herzog (10) require a reliable and locally precise intersubject registration. Whereas the feasibility of this approach seems commonly accepted for brain images $(20,21)$, the same cannot be said for the case of whole-body images, because of the high intersubject variability. The aim of our work was to contribute a method that uses an intersubject registration as input but that can still generate correct predictions in the case of local registration errors.

Determining the attenuation value corresponding to a certain point in an MR image can be regarded as either a regression problem (because the target values are indeed continuously distributed) or, more common in practice, a classification problem. In brain imaging, separation into 3 classes is normally considered to be sufficient, as it is assumed that the histogram of attenuation values has 3 dominant peaks: air, soft tissue, and bone. In whole-body imaging, the lungs are introduced as an additional class, as it is sometimes done with fat. However, on the basis of the histogram of CT values for a scan of the human head (Fig. 1), we do not observe only sharp peaks. The CT histogram corresponding to bone often does not form a sharp peak, because of different bone densities and (unavoidable) partial-volume effects. It would, therefore, be preferential that the attenuation map prediction method also allows for attenuation values on a continuous scale.

Another aim of this work was to develop a method that can reliably predict attenuation values from MR images. We first predict pseudo-CT values on a continuous scale, which are then mapped to the attenuation values as is done in a PET/CT scanner. Our method combines local pattern recognition with atlas registration, to be applicable also to whole-body imaging in which approaches based on registration only are problematic. 
A technical problem in MRI-based AC, in particular for whole-body imaging, is the limited field of view (FOV) of the MRI scanner. For example, in human thorax imaging, the arms of the patient are often not covered. Although the focus of this article is on the AC within the FOV, the atlas registration part of our method could easily be used to obtain predictions outside the tomographic FOV of the MRI scanner.

PET AC has been investigated extensively by numerous authors $(22,23)$. AC is typically performed in 2 steps: first the attenuation map is determined, and then it is applied to the PET emission data during the image reconstruction process, which transforms the sinogram data acquired by the PET scanner via an inverse radon transform to obtain the final PET image. For iterative reconstruction methods, the attenuation map can be used in different ways, as described by Hebert and Leahy (24).

The $\mathrm{AC}$ factor $(\mathrm{ACF})$ for a specific line of response (LOR) relates the measured signal $\mathrm{I}$ to the signal $\mathrm{I}_{0}$ that would be measured in the absence of attenuation:

$$
\mathrm{ACF}_{\mathrm{LOR}}=\frac{\mathrm{I}_{0}}{\mathrm{I}}=\exp \left[\int_{\mathrm{LOR}} \mu^{\mathrm{PET}} \times \mathrm{dx}\right]
$$

Here, $\mu^{\text {PET }}$ is the linear attenuation coefficient for $\mathrm{E}=511-\mathrm{keV}$ photons (25).

Assume that the AC values for each LOR are stored in a matrix ACF such that every entry corresponds to 1 detector pair (i.e., $1 \mathrm{LOR}$ ). The sinogram $\mathrm{SIN}_{\mathrm{ac}}$, which is corrected for photon attenuation, can then be determined by pointwise multiplication of every entry in $\mathrm{ACF}$ with the corresponding entry in the sinogram SIN $_{\text {det }}$ as measured by the PET detectors:

$$
\mathrm{SIN}_{\mathrm{ac}}=\mathrm{SIN}_{\mathrm{det}} \times \mathrm{ACF}
$$

Eq. 2

To use a CT image for PET AC, the CT intensities need to be converted to $511 \mathrm{keV}$, transformed into sinogram space, and exponentiated to yield the ACFs. We introduce methods to predict pseudo-CT images that are not obtained from an actual CT scan. Nevertheless, they share the characteristics of a real CT scan and, therefore, we can subsequently apply the same methods as used on PET/CT scans. We use piecewise linear mapping (5) to convert CT intensities to AC values:

$$
\mu^{\mathrm{PET}}(\mathrm{h})=\left\{\begin{array}{cc}
\mathrm{c} \times(\mathrm{h} / \mathrm{HU}+1,000) & \mathrm{h}<50 \mathrm{HU} \\
\mathrm{b}+\mathrm{a} \times(\mathrm{h} / \mathrm{HU}+1,000) & \mathrm{h}>50 \mathrm{HU}
\end{array}\right.
$$

Eq. 3

where $\mathrm{h}$ is the CT signal intensity, $\mathrm{c}=9.6 \times 10^{-5} \mathrm{~cm}^{-1}$, and $\mathrm{a}$ and $\mathrm{b}$ are constants that depend on the $\mathrm{x}$-ray tube voltages.

AC for simultaneous PET/MRI has received little attention so far. The first simultaneously acquired PET/MR images of the human brain were attenuation-corrected only with 2-class attenuation maps that were derived by thresholding the MR images into tissue versus air (26). The head coil was neglected because its position could not be determined from the MR or PET image.

\section{MATERIALS AND METHODS}

We present 2 different approaches to the problem of pseudo-CT prediction. The first one uses local information, whereas the second one is more global, working with information from the whole image. The two approaches can be combined to obtain a unified method that makes use of both local and global information.

\section{Pattern Recognition with Gaussian Processes}

For all standard MR sequences, the MR intensity of 1 voxel does not contain sufficient information to uniquely determine its tissue class. Figure 2 may, however, motivate the idea that the neighboring voxels surrounding the voxel of interest (VOI), the patch, may add some characteristic information. This may be particularly useful when distinguishing between bone and air because the intensity of the voxel alone is near zero in both cases, but the surrounding volume looks different for air and bone regions.

Pattern recognition methods aim at determining a mapping, $\mathrm{f}: \mathrm{P} \mapsto R$, that maps rectangular $\mathrm{MR}$ image patches, $\mathrm{p}_{\mathrm{i}} \in \mathrm{P}$, centered at the VOI to real-valued CT intensities, $\mathrm{y}_{\mathrm{i}} \in R$. Assuming that a database of coregistered MR/CT image pairs is available, we can automatically (randomly) extract d example pairs, $\left(\mathrm{p}_{\mathrm{i}}, \mathrm{y}_{\mathrm{i}}\right) \in \mathrm{P} \times R$, and use them to train the algorithm, that is, to determine the mapping $f$, which depends on the used MR and CT image characteristics. In particular for the MRI, it is necessary that all images of the database are acquired with the same protocol.

Given the training database, we have a typical regression problem, for which we use gaussian process regression (27). Gaussian processes are examples of kernel machines (28). These methods have been used successfully in a variety of applications (29-31), including prediction on MR images (32), using application-specific kernels. A gaussian process is defined by a mean function, $\mathrm{m}: \mathrm{P} \mapsto R$, and a covariance or kernel function, $\mathrm{k}: \mathrm{P} \times \mathrm{P} \mapsto R$. A nonzero mean function, $\mathrm{m}$, represents knowledge that the target function is close to $\mathrm{m}$. Furthermore, one expects that $\mathrm{CT}$ values corresponding to similar MR patches $\mathrm{p}_{\mathrm{i}}, \mathrm{p}_{\mathrm{j}}$ are highly correlated. The degree of correlation or, equivalently, the similarity of $p_{i}, p_{j}$ is

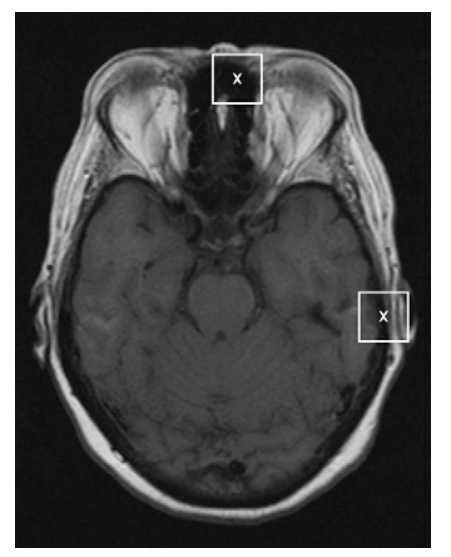

FIGURE 2. Xs indicate 2 voxels in MR image whose tissue classes (air and bone) cannot be distinguished on basis of their intensity alone. If we include surrounding patch (rectangle), we see patterns that are typical for either bone or air, and different attenuation values can be assigned. 
expressed by the kernel function. We used a gaussian function of the form:

$$
\mathrm{k}\left(\mathrm{p}_{\mathrm{i}}, \mathrm{p}_{\mathrm{j}}\right)=\exp \left(-\frac{\left\|\mathrm{p}_{\mathrm{i}}-\mathrm{p}_{\mathrm{j}}\right\|^{2}}{2 \sigma_{\text {patch }}^{2}}\right)
$$

We assume the training labels to be noisy versions of the true labels, for example, because of MRI/CT misalignments. Noisy observations can be encoded into gaussian processes by adding a term, $\sigma_{\mathrm{n}}^{2}$, to the covariance function $\mathrm{k}$ whenever the inputs are identical. For an unseen patch $\mathrm{p}$, gaussian process regression yields a gaussiandistributed predictive distribution for $\mathrm{f}(\mathrm{p})$, with mean and covariance given as:

$$
\begin{gathered}
\mu(\mathrm{p})=\mathrm{m}(\mathrm{p})+\mathrm{k}^{\mathrm{T}} \mathrm{C}_{\mathrm{N}}^{-1}(\mathrm{y}-\mathrm{m}), \\
\sigma^{2}(\mathrm{p})=\mathrm{c}-\mathrm{k}^{\mathrm{T}} \mathrm{C}_{\mathrm{N}}^{-1} \mathrm{k} .
\end{gathered}
$$

Here, $\mathrm{k}$ has elements $\mathrm{k}_{\mathrm{i}}=\mathrm{k}\left(\mathrm{p}_{\mathrm{i}}, \mathrm{p}\right)$ for $\mathrm{i}=1, \ldots, \mathrm{d},\left(\mathrm{C}_{\mathrm{N}}\right)_{\mathrm{i}, \mathrm{j}}=$ $\mathrm{k}\left(\mathrm{p}_{\mathrm{i}}, \mathrm{p}_{\mathrm{j}}\right)+\sigma_{\mathrm{n}}^{2} \delta_{\mathrm{i}, \mathrm{j}}$ for $\mathrm{i}, \mathrm{j}=1, \ldots, \mathrm{d}, \mathrm{c}=\mathrm{k}(\mathrm{p}, \mathrm{p})$, and $\mathrm{m}_{\mathrm{i}}=\mathrm{m}\left(\mathrm{p}_{\mathrm{i}}\right)$.

To predict a pseudo-CT image for a new patient, we extracted for each voxel of the MR image a surrounding patch $p$ and used the mean, $\mu(p)$, of the distribution of $f(p)$ as the best point estimate of the CT value. We did not use the predictive variance $\sigma^{2}(p)$, but it could be used, for example, to reject the pseudo-CT image when the cumulative predictive variance of the whole image is too high. In such a case, one might have to rely on a more primitive prediction method.

\section{MRI/CT Atlas Registration}

Another approach to determine the attenuation map from a given MRI scan of the same patient is with atlas registration. Assume that an atlas comprising an MR image and a corresponding CT image for the same subject is given. Then, for any new subject, a nonrigid registration algorithm could automatically compute a deformation field that aligns the atlas MR image with the new subject's MR image. Applying the same deformation to the atlas CT image would yield the desired result.

Instead of working with just a single image pair as an atlas, we have built an atlas database consisting of MR and CT brain images from patients $(n=17)$. Given a patient MR image, we register all $n$ atlas MR images to the patient MRI, yielding $n$ predictions of the CT value.

To avoid ethical issues related to exposing volunteers to radiation, we used only images from patients who had received MRI and CT scans after clinical indication. Patients were $56 \pm 16 \mathrm{y}$ (5 men, 12 women; sufficient intersubject variance). CT values at corresponding bone positions typically varied by 500 Hounsfield units. For the atlas MR images, a T1-weighted spin-echo (SE) sequence (echo time, $12 \mathrm{~ms}$; repetition time, $500 \mathrm{~ms}$ ) was used. The dimensions of the MRI volumes were $256 \times 192 \times 32$, with a voxel size of $0.89 \times 0.89 \times 4.4 \mathrm{~mm}$. The corresponding CT images were acquired using a tube voltage of $120 \mathrm{kVp}$ and a tube current of $285 \mathrm{mAs}$. The in-plane resolution was $0.43 \times 0.43 \mathrm{~mm}$, and the slice thickness was between 3 and $4.5 \mathrm{~mm}$. The CT images were later resampled to MRI resolution. The necessary intermodality alignment between corresponding MR and CT images in the atlas was performed by first aligning rigidly with MiraView (Siemens Molecular Imaging) using manually placed markers, followed by deformable registration with B-Splines-based implementation (33), using normalized mutual information as a similarity measure. For the registration of the atlas MR images to the patient MR image, we used the spatial normalization function of SPM5 (Wellcome Trust Centre for Neuroimaging). We used standard settings to achieve a registration of all atlas images within approximately 15 min. Although we used human know-how to build the atlas, no manual intervention was necessary at runtime (i.e., to predict a pseudo-CT for a new patient).

\section{Combining Local Pattern Recognition with Atlas Registration}

The approaches described above show practical limitations when used alone. Just using the local patch may fail if the patch describing the neighborhood of the VOI is not characteristic enough for a certain CT value. This might lead to prediction of tissue classes that are highly unlikely to occur at the query position, neglecting global information. For example, this method might predict bone attenuation values in the middle of the brain. On the other hand, it is well known that image-registration methods often do not yield satisfactory results, for example, because of local minima of the nonrigid deformation energy function. More fundamentally, in general we cannot even assume that there exists a one-to-one correspondence between patient and atlas images. For example, it is extremely unlikely that pockets of gas in the abdominal region could be brought into one-to-one correspondence across individuals. In such cases, it may be useful to consider local information around the VOI.

We propose to combine local pattern recognition and atlas registration methods by using atlas registration as prior knowledge, which takes two forms, for the gaussian process regression. First, we suggest using not only the patch but also the registered coordinate of a training point as input. The registered coordinate of a point is its position relative to a coordinate system that is fixed with the anatomy of a reference subject. The motivation for using a registered coordinate is that for a given patch at a certain position in the patient image, the best match in the atlas database is probably found nearby. To be able to compare positions in different images, we have to transform them into a single coordinate system. In our implementation, we chose the reference subject for the registered coordinates to be the patient itself. Thus, we first register all atlas MR images to the patient MR image, applying the same transformations to the corresponding atlas CT images. We then extract training pairs $\left(d_{i}, y_{i}\right)$ from all transformed atlas images, where $d_{i}=\left(p_{i}^{T}, x_{i}^{T}\right)^{T}$ consists of the position $x_{i}$ of the voxel in the patient coordinate system, and $p_{i}$ is the patch surrounding it. Here, $y_{i}$ is the corresponding CT value. Instead of the kernel in Equation 4, we then use:

$$
\mathrm{k}\left(\mathrm{d}_{\mathrm{i}}, \mathrm{d}_{\mathrm{j}}\right)=\exp \left(\frac{-\left\|\mathrm{p}_{\mathrm{i}}-\mathrm{p}_{\mathrm{j}}\right\|^{2}}{2 \sigma_{\text {patch }}^{2}}\right) \times \exp \left(\frac{-\left\|\mathrm{x}_{\mathrm{i}}-\mathrm{x}_{\mathrm{j}}\right\|^{2}}{2 \sigma_{\text {pos }}^{2}}\right) \text {. Eq. } 7
$$

Second, we include prior knowledge of the atlas registration by setting the mean function $\mathrm{m}$ to the average value of the registered $\mathrm{CT}$ images.

A flow chart showing all processing steps that lead from the MR image and the PET detector sinogram to the attenuationcorrected PET image is shown in Figure 3.

The runtime for gaussian-process prediction scales cubically in the number of training data pairs considered. Although this runtime may look prohibitive at first sight, the location-dependent 
kernel reduces this cost. Because the covariance is almost zero for patches that are not in the same area, the patches do not contribute significantly to the prediction. Thus, we have to consider only those training pairs in Equations 5 and 6, which are relatively close to the VOI. This allows us to use many relevant training examples from all the atlas subjects. If necessary, runtimes could be further reduced, for example, by using the predictive variance to adaptively refine a coarse-to-fine multiscale prediction scheme.

All free parameters such as patch size, $\sigma_{\text {patch }}, \sigma_{\text {pos }}$, and $\sigma_{\mathrm{n}}$ were determined using cross-validation. Because the interslice distance of the atlas database was rather large, we constrained the training patches for prediction at a certain point to be extracted from the same image slice only. Typically, we used $9 \times 9$ patches and a 15 -voxel $\sigma_{\text {pos }}$.

\section{Quantitative Evaluation}

Observer interest in predicted pseudo-CT images is not in the display of the images but instead in their application to AC. In the quantitative evaluation of such images, we need to look at how errors in the attenuation map predicted from the MR image propagate to the reconstructed PET image.

In general, a problem arises when evaluating the impact of MRIbased AC (MRAC) versus CT-based AC (CTAC) for the PET images, because MRI, CT, and PET (including the PET sinogram) scans are rarely all available for the same patient. In addition, such low patient numbers might not be sufficient for statistically significant evaluation. Often, only MR and CT images are at hand. To be able to also use these datasets to estimate the impact of MRAC versus CTAC, we use a simulated typical PET image. Therefore, we estimate a typical PET image for each patient and calculate how this PET image would change if it were obtained using MRAC instead of CTAC (in which we use the CT attenuation-corrected image as ground truth).

The process involves the following: The SPM5 toolbox (34) provides template brain MR and ${ }^{18} \mathrm{~F}$-FDG PET images. The MR template image was registered to the patient MR images using SPM5 normalization. The obtained transformations were then applied to the template PET images, thereby yielding PET images for each patient that match the patient anatomy. Next, to obtain PET $_{\text {MRAC }}$ we first need the projection data $\left(\mathrm{SIN}_{\text {det }}\right)$ that are uncorrected for attenuation:

$$
\begin{aligned}
\operatorname{SIN}_{\text {det }} & =\operatorname{Rad}(\mathrm{PET}) / \mathrm{ACF} \\
& =\operatorname{Rad}(\mathrm{PET}) \times \exp \left[-\operatorname{Rad}\left(\mu^{\mathrm{PET}}(\mathrm{CT})\right)\right],
\end{aligned}
$$

where $\mathrm{Rad}$ is the radon transform. Once $\mathrm{SIN}_{\mathrm{det}}$ is obtained, PET $_{\text {MRAC }}$ is calculated by using the following equation:

$$
\begin{aligned}
\operatorname{PET}_{\text {MRAC }}= & \operatorname{Rec}\left(\operatorname{SIN}_{\text {det }} \times \mathrm{ACF}_{\mathrm{MR}}\right) \\
= & \operatorname{Rec}\left(\operatorname{SIN}_{\mathrm{det}} \times \exp \left[\operatorname{Rad}\left(\mu^{\mathrm{PET}}\left(\mathrm{CT}_{\mathrm{MR}}\right)\right)\right]\right) \\
= & \operatorname{Rec}\left(\operatorname{Rad}(\mathrm{PET}) \times \exp \left[\operatorname { R a d } \left(\mu^{\mathrm{PET}}\left(\mathrm{CT}_{\mathrm{MR}}\right)\right.\right.\right. \\
& \left.\left.\left.-\mu^{\mathrm{PET}}(\mathrm{CT})\right)\right]\right)
\end{aligned}
$$

where $\mathrm{CT}_{\mathrm{MR}}$ is the MR-estimated pseudo-CT image and Rec the reconstruction, that is, the inverse radon transform. We therefore have an expression that relates the ground-truth PET and CT images and an estimated pseudo-CT image, $\mathrm{CT}_{\mathrm{MR}}$, with $\mathrm{PET}_{\mathrm{MRAC}}$, the PET image that we obtained by using $\mathrm{CT}_{\mathrm{MR}}$ for the $\mathrm{AC}$.

Differences between PET $_{\text {MRAC }}$ and the original PET image are dependent on the exact reconstruction method used. It is not, however, the focus of this work to compare different reconstruction methods. For the simulated PET images, we have simply reconstructed each 2-dimensional slice with filtered backprojection, neglecting normalization, scatter, and random coincidences, to isolate the bias on emission due to wrong attenuation maps only. We also assume that CT intensities scaled to $511 \mathrm{keV}$ provide the true PET attenuation values.

For the cases in which CT, MR, and PET images were available from the same patient, 2-dimensional ordered-subset expectation maximization with randoms, scatter, and $\mathrm{AC}$ was used to reconstruct the images on the PET/CT scanner. The procedure was performed twice, once with the original CT image and once with the MRderived pseudo-CT image for attenuation and scatter correction.

\section{RESULTS}

We evaluate our method for human brain scans using 2 datasets. First, we use our brain atlas itself to measure the quality of estimated CT images and the impact on the reconstruction of simulated PET images. Second, we eval-

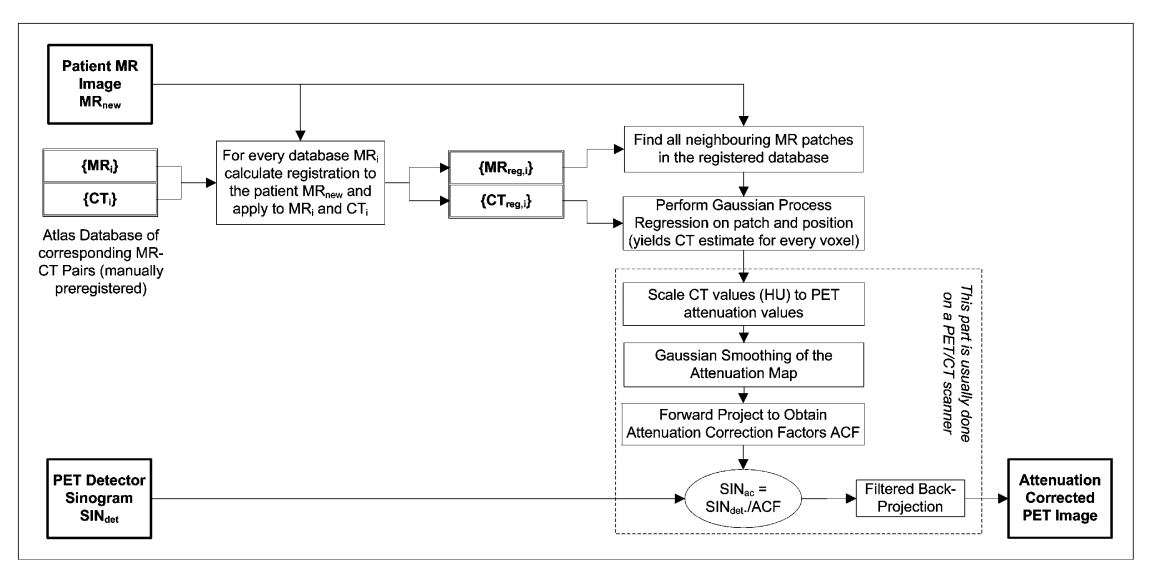

FIGURE 3. Overview of steps involved in our method for obtaining attenuationcorrected PET image, based on PET detector sinogram and MR image. Resampling of MRI, PET, and CT to required resolution is performed wherever necessary. 
uate our method on $3 \mathrm{MR} / \mathrm{PET} / \mathrm{CT}$ datasets that are independent of the atlas dataset. Last, we give an outlook of whole-body imaging with a rabbit dataset.

\section{Evaluation of the Atlas Database}

We used leave-one-out cross-validation (LOOCV) to predict pseudo-CT images for every MR image, using the remaining $n=16$ image pairs as a reduced atlas. The average absolute LOOCV error per voxel of the predicted pseudo-CT images was 100.7 Hounsfield units. As can be seen in Figure 4, even in regions in which bone and air are mixed, our method is able to distinguish bone, air, and soft tissue with high accuracy.

As described in an earlier section, we generated simulated PET images for each MR image in the atlas database and then compared our MRAC method with the CTAC method on these images. Figure 5 shows the joint histograms of the PET images obtained with the different AC schemes and the CT attenuation-corrected PET image that served as ground truth.

Regression line coefficients and $R^{2}$ values were calculated for all 17 patients, using linear regression with the expression $y=\alpha+\beta x$, where $x$ is the ground-truth PET image and $\mathrm{y}$ is a reconstruction based on one of the presented AC schemes. Results are shown in Table 1.

The 2-class AC was implemented using an inside/outside model. The inside of the patient was determined using a series of morphologic operations. Visual inspection confirmed that the method determined the inside with high accuracy. Voxels inside the patient were assigned a uniform attenuation coefficient of $0.096 \mathrm{~cm}^{-1}$.

As expected, nonperforming AC leads to image intensities that deviate from the true image by factors of up to around 10

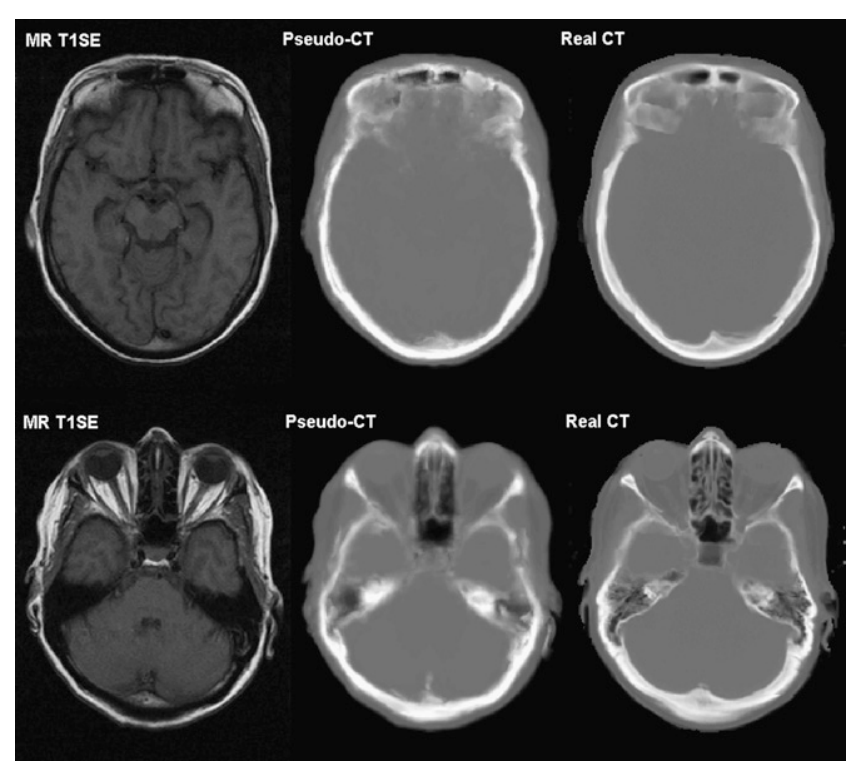

FIGURE 4. Images from patient's T1-weighted spin-echo MRI (left), pseudo-CT (as predicted using our method; middle), and real CT (right) scans. MR T1SE = T1-weighted spin-echo MRI. and are therefore not useful for quantitative analysis. Twoclass AC, which ignores bone, leads to systematic underestimation of activity and also to high variance. The proposed method for AC has no significant over- or underestimation of activity and has low statistical variance.

\section{Evaluation of MRI/PET/CT Triplets}

For 3 patients, who had both PET/CT and MR scans of the head, we validated our method by rerunning the PET reconstruction on the scanner (16 HiRez; Siemens Biograph), using our MRI-estimated pseudo-CT image instead of the original CT scan for scatter and AC.

In a combined PET/MRI scanner, the registration of the PET and MR image will be a trivial issue because the PET and the MRI gantry are physically aligned relative to each other. Currently, in the absence of a clinically usable combined PET/MRI scanner, we were still faced with the issue of how to align the MR image, and the attenuation image derived from it, with the emission data. Accurate alignment of the attenuation image to the emission data is crucial for accurate AC. However, this alignment step is not a focus of this article; we simply aligned the pseudo-CT images with the available CT images, using the automatic rigid registration function in MiraView.

Outside the MRI scanner FOV, we did not predict pseudo-CT values but used the intensities from the original CT image instead. For the subsequent analysis, we ensured that we analyzed only the effect of MRI-based AC in slices in which the complete cross-section of the subject body was inside the FOV of the MRI. We ignored the possible impact of the attenuation and scatter of the MRI coil that would be present in a real PET/MRI scanner.

Figure 6 shows 1 slice of the PET and CT images obtained on the PET/CT scanner and the corresponding pseudo-CT and PET images obtained with MRI-based AC. Although the difference between the CT and pseudo-CT image is visible, the effect on the PET images is relatively small and the visual impression is that the PET images are similar. Quantification was done by a nuclear medicine expert who drew regions of interest (ROIs). For the 2 patients who underwent ${ }^{18} \mathrm{~F}$-FDG PET scans, 12 ellipses were placed in the dorsal cortex, frontal cortex, lateral cortex, caudate nucleus, thalamus, and white matter, each time left and right. The third patient underwent a ${ }^{68} \mathrm{Ga}$-DOTATOC (where DOTA = tetraazacyclododecane tetraacetic acid and TOC $=$ D-Phe-c(Cys-TyrD-Trp-Lys-Thr-Cys)-Thr(ol)) PET scan, and 50\% isocontour lines were drawn on 3 regions with increased uptake (2 lesions and the normal hypophysis).

For the ROIs of all 3 patients, the mean percentage difference in activity between PET $_{\text {CTAC }}$ and PET $_{\text {MRAC }}$ was $3.2 \% \pm 2.5 \%$. Only for 1 ROI did we observe a problematic quantification error, an activity overestimation of $10 \%$. The patient examined with DOTATOC presented with a small Falx meningioma positioned directly next to the skull, and our pseudo-CT overestimated the bone thickness in this slice. The meningioma now appeared as if partly embedded 


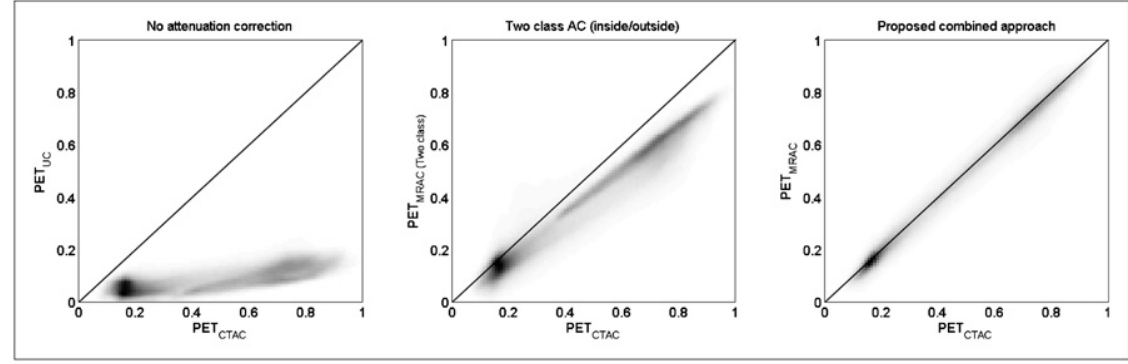

FIGURE 5. Joint histograms showing no $A C(A)$, simple $A C$ using attenuation map with only 2 attenuation values $(B)$, and $\mathrm{AC}$ using our MRI-based predicted attenuation map (C). No significant systematic over- or underestimation of activity is demonstrated. in the bone, which led to increased $\mathrm{AC}$ and hence the relatively high quantification error at the location of the lesion.

\section{Initial Results on Whole-Body Images}

To apply our method to whole-body AC, we have obtained MRI and CT scans from several euthanized rabbits. However, despite our best efforts to minimize movement between the scans by using a portable bed pallet, it has proved difficult to obtain well-registered MRI/CT pairs. For 2 rabbit datasets, the MR and the CT image could be aligned with sufficient accuracy, which is why we present only preliminary results for whole-body AC.

Given the $2 \mathrm{MRI} / \mathrm{CT}$ rabbit datasets, we used 1 rabbit as the atlas and predicted the CT image of the second rabbit, the "patient," from its MR image. Because the training database in this case consisted of only 1 example, we did not try to predict a real-value CT image but confined ourselves to the simpler task of predicting merely 3 classes: bone, soft tissue, and air. This multiclass prediction was performed using a one-versus-rest support vector machine (28). Note that support vector machines are structurally similar to gaussian processes, because the key design element is again a positive definite kernel, for which we used the one given in Equation 7. Also, because the intersubject registration for whole-body images is far more difficult than that for brain images, we have used a simple automatic rigid registration (MiraView) to align the MR images of the atlas and the patient.

Figure 7 shows the results of this 3-class classification, compared with thresholding of the CT image. The figure shows that reasonable prediction quality can be achieved, even with only a crude rigid alignment.

TABLE 1

Regression Coefficients and $R^{2}$ Values

\begin{tabular}{lrcr}
\hline & \multicolumn{3}{c}{$R^{2}$ value for... } \\
\cline { 2 - 4 } Coefficient & $\begin{array}{c}\text { Proposed } \\
\text { method }\end{array}$ & $\begin{array}{c}\text { Uncorrected } \\
\text { images }\end{array}$ & $\begin{array}{c}\text { Two-class } \\
\text { AC images }\end{array}$ \\
\hline$\alpha$ & $-0.006 \pm 0.007$ & $0.025 \pm 0.004$ & $0.014 \pm 0.009$ \\
$\beta$ & $0.990 \pm 0.026$ & $0.138 \pm 0.019$ & $0.78 \pm 0.030$ \\
$R^{2}$ & $0.968 \pm 0.011$ & $0.476 \pm 0.076$ & $0.884 \pm 0.044$ \\
\hline
\end{tabular}

\section{DISCUSSION}

On a dataset of MRI/CT brain images from 17 patients, we have shown that our method enables, solely based on MRI information, the prediction of pseudo-CT images that are similar to the actual CT images. We have simulated PET images and attenuated sinograms for these 17 patients and calculated that the impact of using the pseudo-CT images instead of the actual CT images for AC is small.

On an additional dataset of PET/CT and MR images from 3 patients, we have rerun reconstructions on the PET/CT scanner, using our pseudo-CT images for AC. Again the similarity of pseudo-CT images to actual CT images was good and PET quantification similar to the CT attenuationcorrected images. In one case, there were 2 confounding effects. The lesion was adjacent to the skull, and the thickness of the skull at that point was overestimated. Even in this pathologic situation, the PET quantification error was approximately only $10 \%$.

The question arises as to how large quantification errors can be and still be acceptable before the error might influence diagnosis. This question has been studied previously $(15,35)$, but in general it is difficult to answer: It depends, among other things, on the body organ, the type of disease that one is trying to diagnose, and even on the experience of the physician. Judging from our personal communications with nuclear

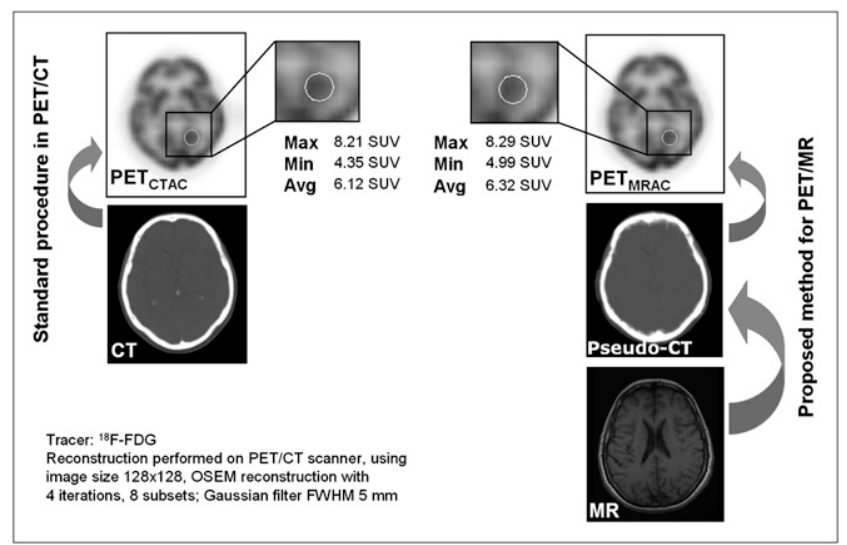

FIGURE 6. Direct comparison of CT attenuation-corrected PET image and PET image that was obtained with AC based on pseudo-CT that was calculated from MR image only. Maximum (Max), minimum (Min), and average (Avg) standardized uptake values (SUV) are given for shown ROI. OSEM = ordered-subset expectation maximization; FWHM = full width at half maximum. 
A

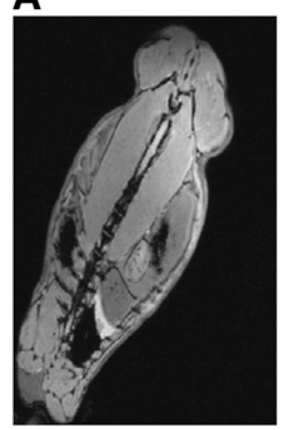

B
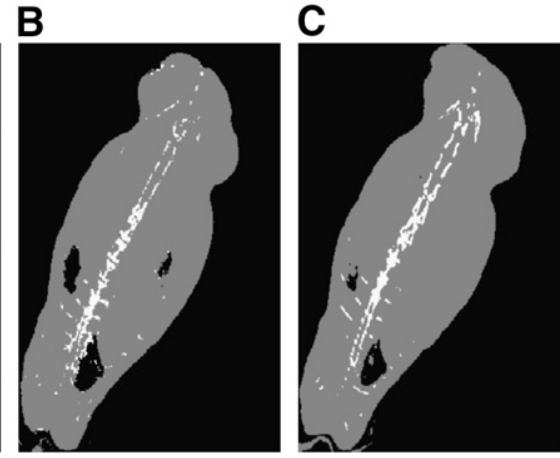

FIGURE 7. (A) MRI T2-weighted multi-echo data-imaging combination of rabbit. (B) Three-class labels as predicted using simple variation of our method for 3-class classification. (C) Three-class labels obtained by thresholding of CT image of rabbit. Not all differences between $B$ and $C$ are because of false predictions. Despite best efforts to physically fixate rabbit between MRI and CT scans, there was still some slight movement between scans, which explains misalignment between $\mathrm{A}$ and $\mathrm{C}$.

medicine experts, there seems to be agreement that quantification errors of $10 \%$ or less typically do not affect diagnosis.

Our approach is not limited to brain imaging. Instead, we assume that our method is particularly strong for wholebody AC. The initial results are promising; however, we do not yet have sufficient data to verify this assumption.

One possibility to further improve the quantitative accuracy of our method would be to try different MRI sequences, in particular, those sequences that might allow a more precise delineation of bone or better estimation of bone density. The impact of the MRI sequence choice might be higher with particular bone-optimized sequences such as ultrashort echo time MRI. However, in whole-body imaging, problems such as tomographic FOV truncation cannot be tackled by such a sequence alone, and again a combination of local classification and an atlas-based method would probably remain the solution of choice. An additional possibility for attenuation map prediction outside the FOV would be to use the emission data as input.

A further improvement can be expected with a higher number of well-aligned CT and MRI training datasets. However, the acquisition of such image pairs turned out to be difficult, especially for whole-body imaging. This is because of unavoidable patient movement between the MRI and the CT scans. Additionally, magnetic field inhomogenities lead to geometric distortions in the MR image. The pattern recognition part can perform only as well as the data that are used from training. We are currently working on the acquisition of human whole-body MRI and CT scans.

\section{CONCLUSION}

A novel method for attenuation map prediction based on the MR image has been presented. It has been evaluated for brain imaging on a dataset of $17 \mathrm{MRI} / \mathrm{CT}$ pairs and on an additional dataset of $3 \mathrm{MRI} / \mathrm{PET} / \mathrm{CT}$ triplets. The results show that MRI-based AC of PET images is possible with high accuracy. The proposed method does not rely on a high local accuracy of the atlas registration and therefore seems particularly promising for whole-body applications, supported by initial results from animal whole-body scans.

\section{ACKNOWLEDGMENTS}

We thank Horst Bock, Michael Erb, Henriette Hehners, Martin Judenhofer, Christiane Lindner, Stephen Lokitz, Niklas Rehfeld, and Hans Wehrl for help with data acquisition. We are also grateful to John Ashburner for helpful feedback.

\section{REFERENCES}

1. Catana C, Wu Y, Judenhofer MS, Qi J, Pichler BJ, Cherry SR. Simultaneous acquisition of multislice PET and MR images: initial results with a MRcompatible PET scanner. J Nucl Med. 2006;47:1968-1976.

2. Judenhofer MS, Catana C, Swann BK, et al. Simultaneous PET/MR images, acquired with a compact MRI compatible PET detector in a 7 tesla magnet. Radiology. 2007;244:807-814.

3. Judenhofer MS, Wehrl HF, Newport DF, et al. Simultaneous PET/MRI: a new perspective for functional and morphological imaging. Nat Med. 2008;14:459465.

4. Carney JP, Townsend DW, Rappoport V, Bendriem B. Method for transforming CT images for attenuation correction in PET/CT imaging. Med Phys. 2006; 33:976-983

5. Beyer M, Weigert M, Quick HH, et al. MR-based attenuation correction for torso-PET/MR imaging: pitfalls in mapping MR to CT data. Eur J Nucl Med Mol Imaging. 2008;35:1142-1146.

6. Sijbers J, Scheunders P, Verhoye M, van der Linden A, van Dyck D, Raman E. Watershed-based segmentation of 3D MR data for volume quantization. Magn Reson Imaging. 1997;15:679-688.

7. Gonzalez RC, Woods RE. Digital Image Processing. Upper Saddle River, NJ: Prentice Hall; 2002.

8. Zhang Y, Brady JM, Smith S. Segmentation of brain MR images through a hidden Markov random field model and the expectation-maximization algorithm. IEEE Trans Med Imaging. 2001;20:45-57.

9. Martinez-Möller A, Souvatzoglou M, Botnar R, et al. An approach for MR-based attenuation correction for combined MR/PET: effects of ignoring bones [abstract]. J Nucl Med. 2007;48 (suppl 2):156P.

10. Kops ER, Herzog H. Alternative methods for attenuation correction for PET images in MR-PET scanners. 2007 IEEE Nucl Sci Symp Conf Rec. 2007;6:4327-4330.

11. Zaidi H. Is MR-guided attenuation correction a viable option for dual-modality PET/MR imaging? Radiology. 2007;244:639-642.

12. Le Goff-Rougetet R, Frouin V, Mangin JF, Bendriem B. Segmented MR images for brain attenuation correction in PET. Proceedings of SPIE. 1994:725-736.

13. El Fakhri G, Kijewski MF, Johnson KA, Syrkin G, Killiany RJ. MRI-guided SPECT perfusion measures and volumetric MRI in prodromal Alzheimer disease. Arch Neurol. 2003;60:1066-1072.

14. El Fakhri G, Kijewski MF, Moore SC. Absolute activity quantitation from projections using an analytical approach: comparison with iterative methods in Tc-99m and I-123 brain SPECT. IEEE Trans Nucl Sci. 2001;48:768-773.

15. Zaidi H, Montandon ML, Meikle S. Strategies for attenuation compensation in neurological PET studies. Neuroimage. 2007;34:518-541.

16. Zaidi H, Montandon ML, Slosman DO. Magnetic resonance imaging-guided attenuation and scatter corrections in three-dimensional brain positron emission tomography. Med Phys. 2003;30:937-948.

17. Dogdas B, Shattuck DW, Leahy RM. Segmentation of skull and scalp in 3-D human MRI using mathematical morphology. Hum Brain Mapp. 2005;26:273-285.

18. Kops ER, Qin P, Müller-Veggian M, Herzog H. MRI based attenuation correction for brain PET images. Springer Proc in Phys. 2007;114:93-97.

19. Robson MD, Bydder GM. Clinical ultrashort echo time imaging of bone and other connective tissues. NMR Biomed. 2006;19:765-780.

20. Friston KJ, Ashburner J, Frith CD, Poline JB, Heather JD, Frackowiak RSJ. Spatial registration and normalization of images. Hum Brain Mapp. 1995;2:1-25.

21. Jenkinson M, Smith S. A global optimisation method for robust affine registration of brain images. Med Image Anal. 2001;5:143-156. 
22. Kinahan PE, Townsend DW, Beyer T, Sashin D. Attenuation correction for a combined 3D PET/CT scanner. Med Phys. 1998;25:2046-2054.

23. Chow PL, Rannou FR, Chatziioannou AF. Attenuation correction for small animal PET tomographs. Phys Med Biol. 2005;50:1837-1850.

24. Hebert TJ, Leahy R. Fast methods for including attenuation in the EM algorithm. IEEE Trans Nucl Sci. 1990;37:754-758.

25. Saha GB. Basics of PET Imaging. New York, NY: Springer; 2005.

26. Schlemmer H-P, Pichler BJ, Wienhard K, et al. Simultaneous MR/PET for brain imaging: first patient scans [abstract]. J Nucl Med. 2007;48(suppl 2):45P.

27. Rasmussen CE, Williams CKI. Gaussian Processes for Machine Learning. Cambridge, MA: MIT Press; 2006.

28. Schölkopf B, Smola A. Learning with Kernels: Support Vector Machines, Regularization, Optimization, and Beyond. Cambridge, MA: MIT Press; 2002.

29. Burckin T, Nagel R, Mandel-Gutfreund Y, et al. Exploring functional relationships between components of the gene expression machinery. Nat Struct Mol Biol. 2005;12:175-182.
30. Noble WS. What is a support vector machine? Nat Biotechnol. 2006;24:15651567.

31. Loo LH, Wu LF, Altschuler SJ. Image-based multivariate profiling of drug responses from single cells. Nat Methods. 2007;4:445-453.

32. Klöppel S, Stonnington CM, Chu C, et al. Automatic classification of MR scans in Alzheimer's disease. Brain. 2008;131:681-689.

33. Ibanez L, Schroeder W, Ng L, Cates J. The ITK Software Guide. 2nd ed. Clifton Park, NY: Kitware, Inc.; 2005. Available at: http://www.itk.org/ItkSoftwareGuide. pdf. Accessed September 2, 2008.

34. Friston K, Ashburner J, Heather J, Holmes A, Poline JB. Statistical Parametric Mapping (SPM5). London, England: The Wellcome Department of Cognitive Neurology, University College London; 2005. Available at: http://www.fil.ion. ucl.ac.uk/spm. Accessed September 2, 2008.

35. Bai C, Kinahan PE, Brasse D, et al. An analytic study of the effects of attenuation on tumor detection in whole-body PET oncology imaging. $\mathrm{J} \mathrm{Nucl}$ Med. 2003;44:1855-1861. 